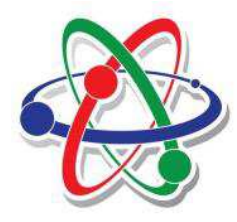

Open Medscience

Peer-Reviewed Open Access

JOURNAL OF DIAGNOSTIC IMAGING IN THERAPY

Journal homepage: www.openmedscience.com

Research Article

\title{
Polymeric nano-hydroxyapatite coated with polylactic acid (PLA): considering new possibilities for radiopharmacy
}

\section{Marta de Souza Albernaz ${ }^{1}$, Gilberto Weissmuller ${ }^{2}$, Andre Linhares Rossi ${ }^{3}$, Alexandre Malta Rossi $^{3}$, Ralph Santos-Oliveira ${ }^{4, *}$}

${ }^{1}$ University Hospital Clementino Fraga Filho-Federal University of Rio de Janeiro, Brazil

${ }^{2}$ Universidade Federal do Rio de Janeiro-Instituto de Biofísica Carlos Chagas Filho, Rio de Janeiro, Brazil

${ }^{3}$ Brazilian Center for Physics Research, Rio de Janeiro, Brazil

${ }^{4}$ Zona Oeste Estadual University, Laboratory of Radiopharmacy, Rio de Janeiro, Brazil; Laboratory of Nanoradiopharmaceuticals, Brazilian Association of Radiopharmacy, Rio de Janeiro, Brazil

*Author to whom correspondence should be addressed:

Ralph Santos-Oliveira, Ph.D.

Laboratory of Nanoradiopharmaceuticals

ralpholiveira@uezo.rj.gov.br

\begin{abstract}
The use of nanotechnology - especially in the area of human health - is increasing every day, with the application of various materials such as hydroxyapatite being amongst the most studied. Consequently, the affinity of hydroxyapatite compatible with so many applications in the human body is evolving cutting edge technology - the result of which is nanoparticles. However, despite these important
\end{abstract}


developments in nanotechnology, encapsulating a nano-hydroxyapatite is still at an early stage of development warranting further investigation.

In this article, we describe a successful method which uses polylactic acid as the polymer to encapsulate nano-hydroxyapatite: this culminates in new applications for oncology and radiopharmacy. In essence, it is the ability to link a radionuclide and /or other substances, e.g. aptamers to enable the creation of new nanoparticle(s) thereby providing novel structural features which are adjuvant to its conventional use.

Key words: radiopharmaceuticals; nanotechnology; oncology; atomic force microscopy; nanohydroxyapatite

\section{Introduction}

The design and development of a biomaterial that is able to replace the form and function of native tissue - whilst promoting regeneration without the onset of necrosis or scar formation - is a challenging area of research. There are many unique properties of nanomaterials: such as increased wettability and surface area, leading to increased protein adsorption, when compared with conventional biomaterials. Cell-scaffold interactions at the cell-material nanointerface may be mediated by integrin-triggered signalling pathways, which affect cell behaviour [1]. Studies have indicated that nano-hydroxyapatite (n-HA) has excellent biological performance and remains a potential candidate to be used as a bioactive material for bone tissue repair [2].

Nonetheless, the extensive application of n-HA is still limited because of its powder form and brittle nature. Biodegradable polymers and their co-polymers have been investigated widely and used for bone regeneration, dental repair, orthopedic fixation devices amongst other biomedical applications $[3]$.

\section{Materials and Methods}

\section{Hydroxyapatite}

The hydroxyapatite was precipitated by a dropwise addition of $\left(\mathrm{NH}_{4}\right)_{2} \mathrm{HPO}_{4}$ aqueous solution containing $\mathrm{NH}_{4} \mathrm{OH}$, to a solution of $\mathrm{Ca}\left(\mathrm{NO}_{3}\right)_{2}$ at $37^{\circ} \mathrm{C}$ and $\mathrm{pH}$ equal to 11 . The precipitate was separated by filtration, repeatedly washed with deionized boiling water and dried at $100^{\circ} \mathrm{C}$ for 24 hours. The dried powder was manually grounded and the $<210 \mu \mathrm{m}$ particles separated by sieving.

Calcium and phosphorous concentrations $(\mathrm{Ca} / \mathrm{P}=1.66)$ were determined by $\mathrm{X}$-ray fluorescence spectroscopy. The sample mineral phase and crystallinity was evaluated by X-ray diffractometry (XRD) with $\mathrm{CuK}$ radiation at $40 \mathrm{kV}$ and $40 \mathrm{~mA}$. 
The phosphate species and $\mathrm{OH}$ - groups in the apatite structure were identified by Fourier transform infrared spectrophotometry in transmission mode from 400 to $4000 \mathrm{~cm}^{-1}$. Crystallite mean size $(\tau)$ along hydroxyapatite (002) and (300) directions was determined by the Debye-Sherrer formula:

$$
\tau=\frac{K \lambda}{\beta_{1 / 2} \cos \theta}
$$

where $\beta_{1 / 2}$ is the peak line width (values in radians) of the reflection and $K=0.9$.

\section{Coating}

$0.20021 \mathrm{~g}$ of nano-hydroxyapatite $(10 \% \mathrm{w} / \mathrm{v})$ was mixed with $0.002 \mathrm{~g}$ of PVA (polyvinyl acid $85 \%$ hydrolyzed, Sigma-Aldrich, $0.1 \% \mathrm{w} / \mathrm{v})$. To this mixture was added a solution of $2 \mathrm{~mL}$ of dichloromethane containing $0.0503 \mathrm{~g}$ of polylactic acid (PLA 40-100 $\mathrm{kDa}$ viscosity from 0.15 to 0.25 , Sigma-Aldrich). This mixture was then ultrasonicated for $2 \mathrm{~min}$ at $55 \mathrm{~W}$ (potency) then $4 \mathrm{~mL}$ of an aqueous solution of PVA $0.028 \mathrm{~g}(0.7 \% \mathrm{w} / \mathrm{v})$ was added. The final mixture was emulsified for 2 cycles for $2 \mathrm{~min}$ at $55 \mathrm{~W}$. Finally, the dichloromethane was evaporated under vacuum at $37^{\circ} \mathrm{C}$ for $20 \mathrm{~min}$. The nanoparticles were recovered and washed with MiliQ water three times by centrifugation at 20,238 x $\mathrm{g}$ for 10 minutes at ambient temperature.

\section{Atomic Force Microscopy}

The emulsion (obtained by step encapsulating), was diluted using 9 parts of MiliQ water and $5 \mu \mathrm{L}$ of the diluted sample and poured into freshly cleaved mica and then dried for 3 minutes with nitrogen gas. The images were created on the Fast-Scan AFM, with silicon cantilever, $\mathrm{k}=0.8 \mathrm{nN}$ at peak force using the model QNM®.

\section{Scanning Electron Microscopy (SEM)}

The morphology of the hydroxyapatite nanoparticles was examined by scanning electron microscopy (SEM) (JEOL LSM 5800). The samples were sputter coated with a layer of gold for observation at 10 $\mathrm{kV}$.

\section{Transmission Electron Microscopy (TEM)}

Micrographs were recorded using a JEOL transmission electron microscope (TEM) model JEM-2010 with a LaB6 filament as the electron source, operated at $200 \mathrm{kV}$. Material samples were mounted on a microgrid carbon polymer and supported on a copper grid, by placing a few droplets of a suspension of the sample in water, followed by drying at ambient temperature. 


\section{Results and Discussion}

The images obtained from using atomic force microscopy, show the formation of spherical structures with size ranging from 100 to $800 \mathrm{~nm}$. These structures must be covered with more than one nanostructure of hydroxyapatite and consequently there was a large variation in size (Figure 1).

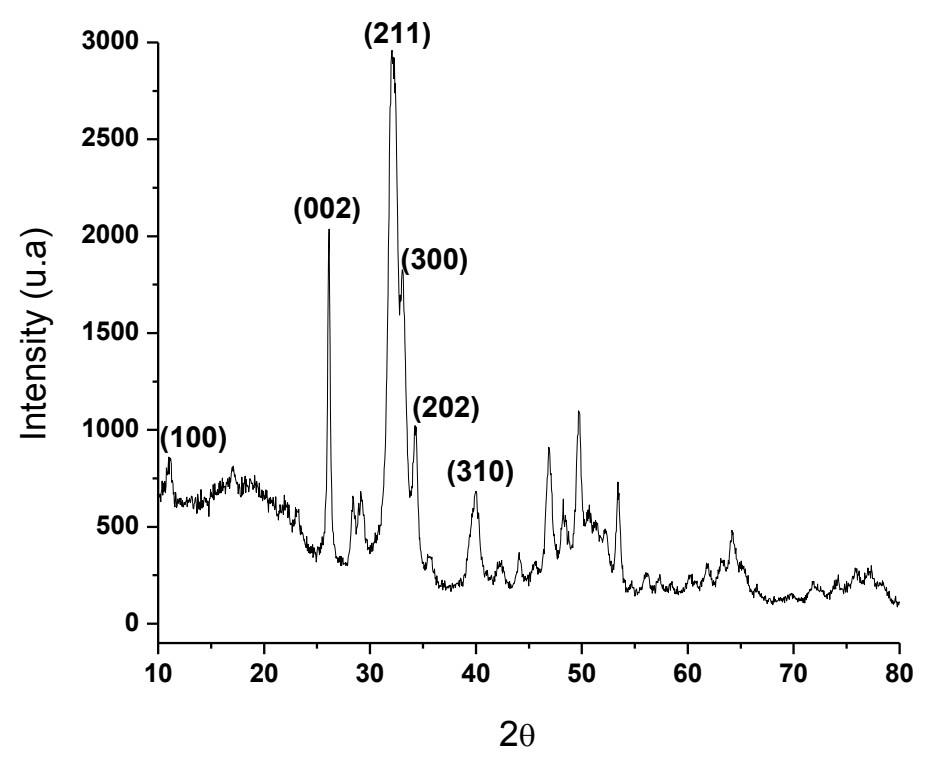

Figure 1. X-ray diffraction pattern of nanostructured hydroxyapatite.

These structures correspond to polymeric nanoparticles of PLA/PVA/nano-hydroxyapatite. In the image (Figure 2), it is possible to observe a cluster in the top left corner resembling a cluster of powdered nano-hydroxyapatite which has not been encapsulated during the process.

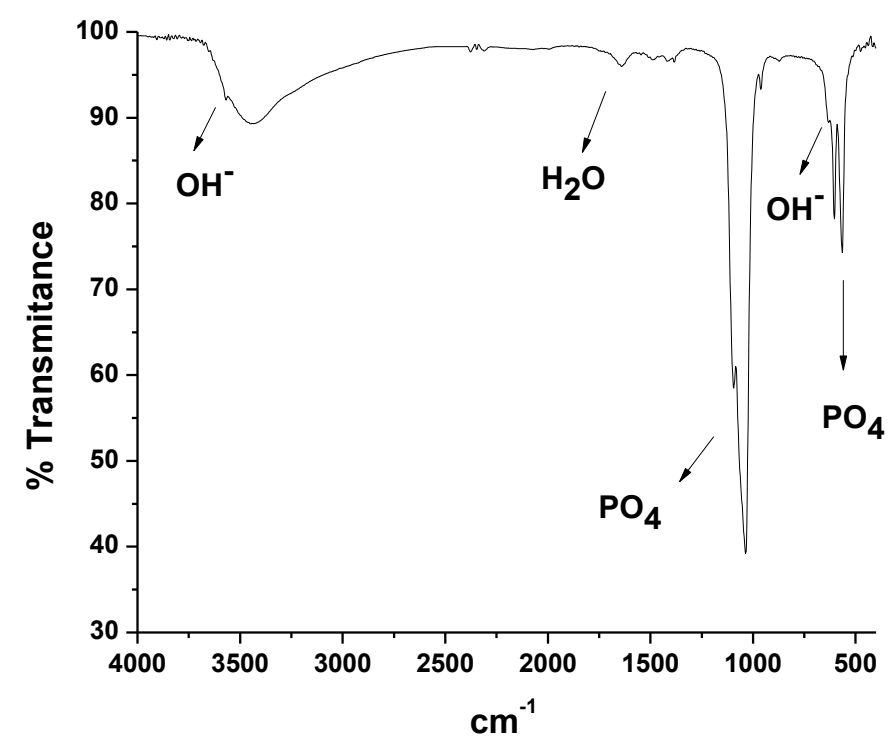

Figure 2. FTIR spectrum of hydroxyapatite sample showing $\mathrm{OH}^{-}$and $\mathrm{PO}_{4}{ }^{3-}$ bands. 
Since nano-hydroxyapatite probably lost its structure and functionality during the encapsulation process due to high sonication. Also, in Figure $\mathbf{2}$ it is possible to observe that the polymeric nanoparticles did not present a smooth surface and it is possible to see a few protuberances.

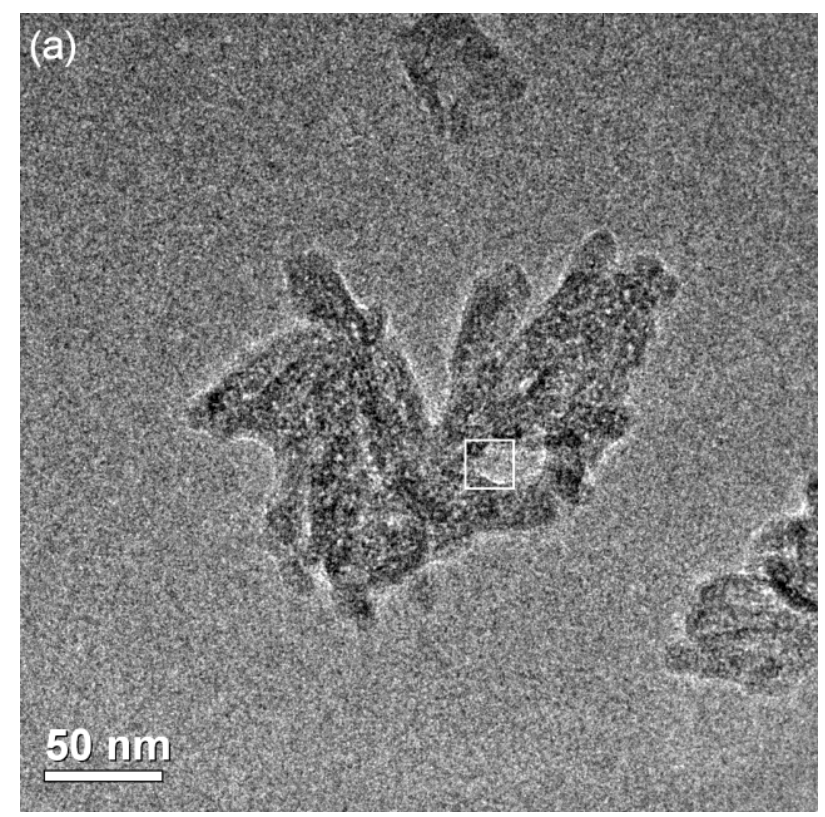

Figure 3. a) Transmission Electron Microscopy (TEM) image of the HA37 sample showing agglomerates of nanoparticles.

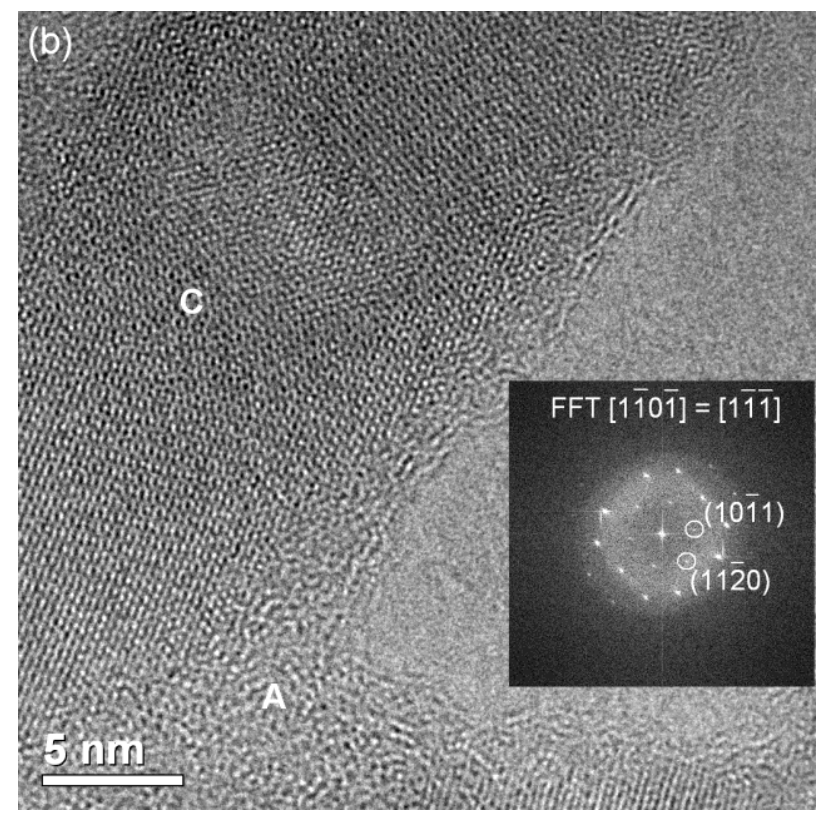

Figure 3. b) High resolution TEM image (HRTEM) of the framed area with its respective FFT along the hydroxyapatite [1101] zone axis.

ISSN: 2057-3782 (Online) 
In Figure 3, it is possible to observe the adhesion of the polymeric nano-hydroxyapatite. This image indicates that darker regions are composed of materials with lower adherence by the cantilever and the opposite is observed in lighter regions. Two more images were made to confirm these observations (Figure 4 and 5).

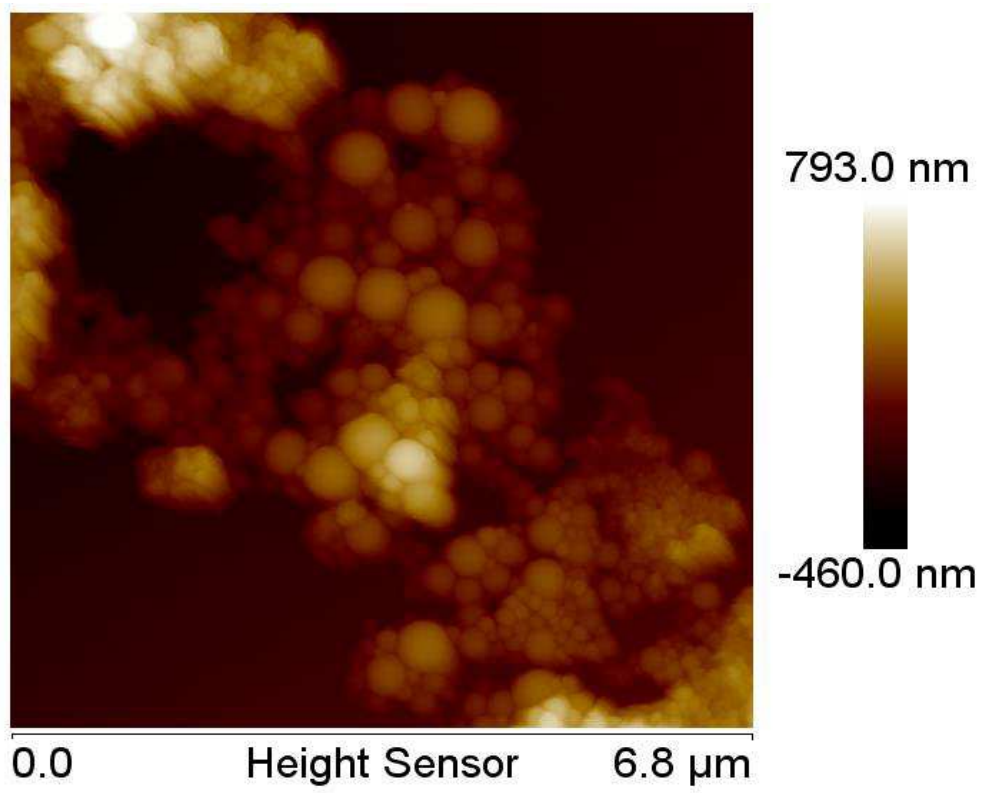

Figure 4. AFM topographic from the nano-hydroxyapatite with PLA/PVA.

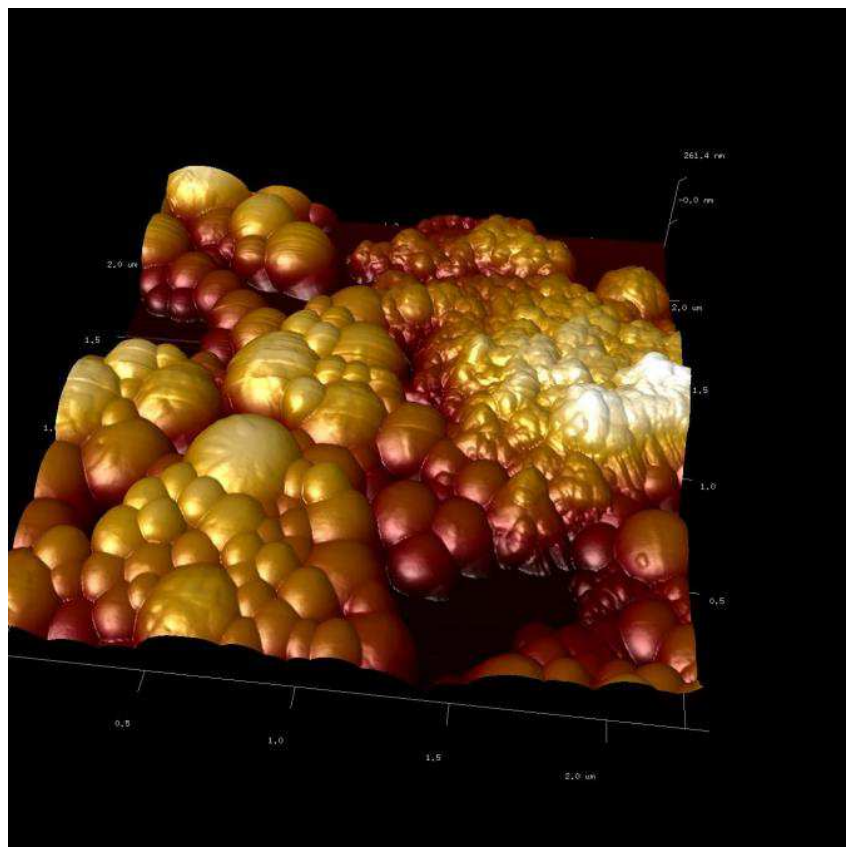

Figure 5. AFM showing the two structures. The first one shows agglomerates and the second one indicates spherical nanoparticles.

ISSN: 2057-3782 (Online) 
Our studies can confirm that the dust agglomerates have lower adhesion than the polymeric nanoparticles. Moreover, the polymeric particles - by their composition of different polymers - have regions with different adhesions to the cantilever. This concludes that polymeric nano-hydroxyapatite have been formed (Figure 6, 7 and 8). The nanoparticles of PLA/PVA/nano-hydroxyapatite were analyzed by TEM.

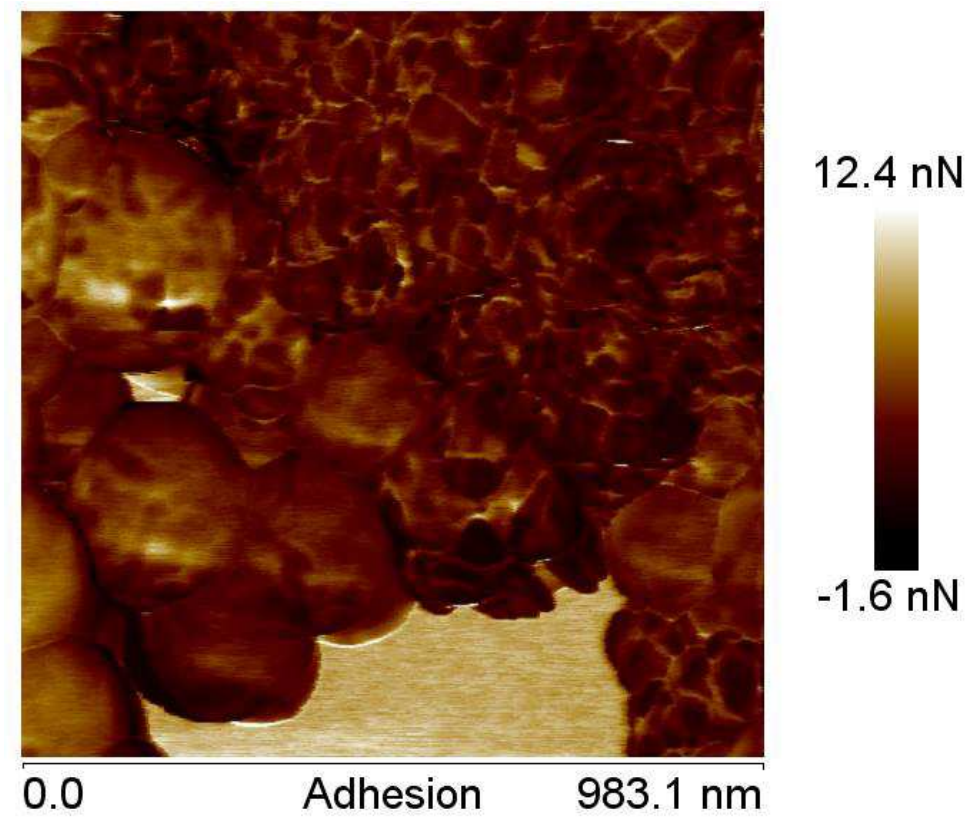

Figure 6. AFM adhesion image, showing the difference between the components of the nanoparticles, confirming the coating of the nano-hydroxyapatite.

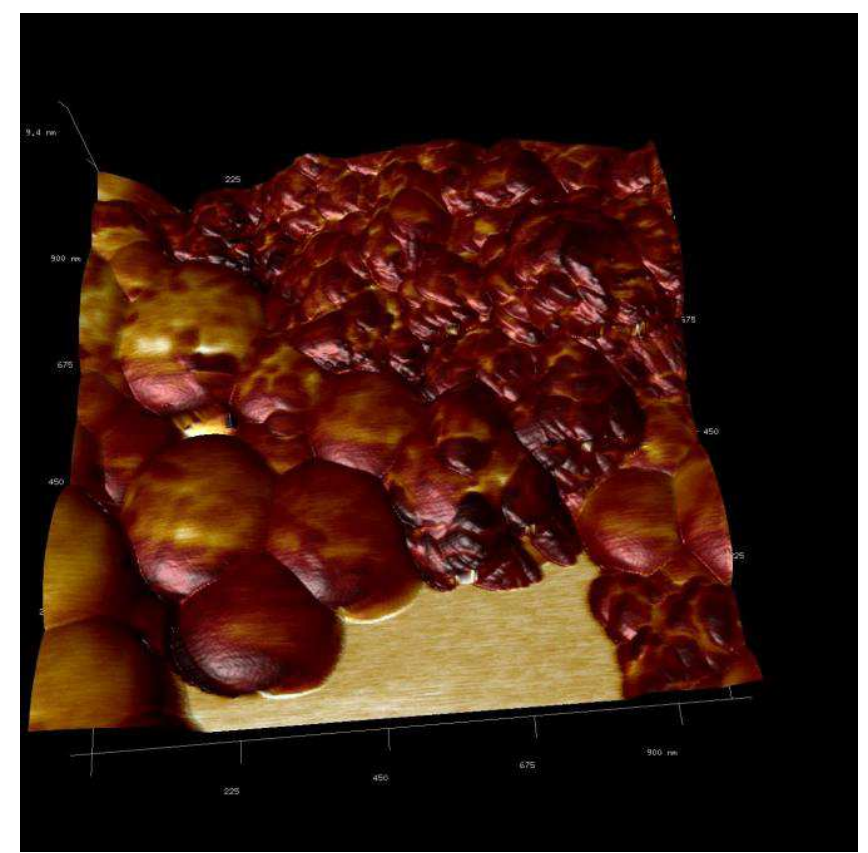

Figure 7. AFM overlay imaging. It is possible to observe the difference between the adhesion of the different materials of the nanoparticle.

ISSN: 2057-3782 (Online) 
In Figure 7, HA crystals (which have more electron-dense particles) were observed associated to polymers (less electron-dense regions). Select area showing electron diffraction from the HA crystals indicated (211) and (002) planes of hydroxyapatite, which corresponded to $0.28 \mathrm{~nm}$ and $0.34 \mathrm{~nm}$ respectively.

In addition it is possible to observe the adhesion difference by observing the dust (agglomerates) and polymeric nanoparticle. In the majority of cases HA crystals were partially encapsulated by the polymer (Figure 8).

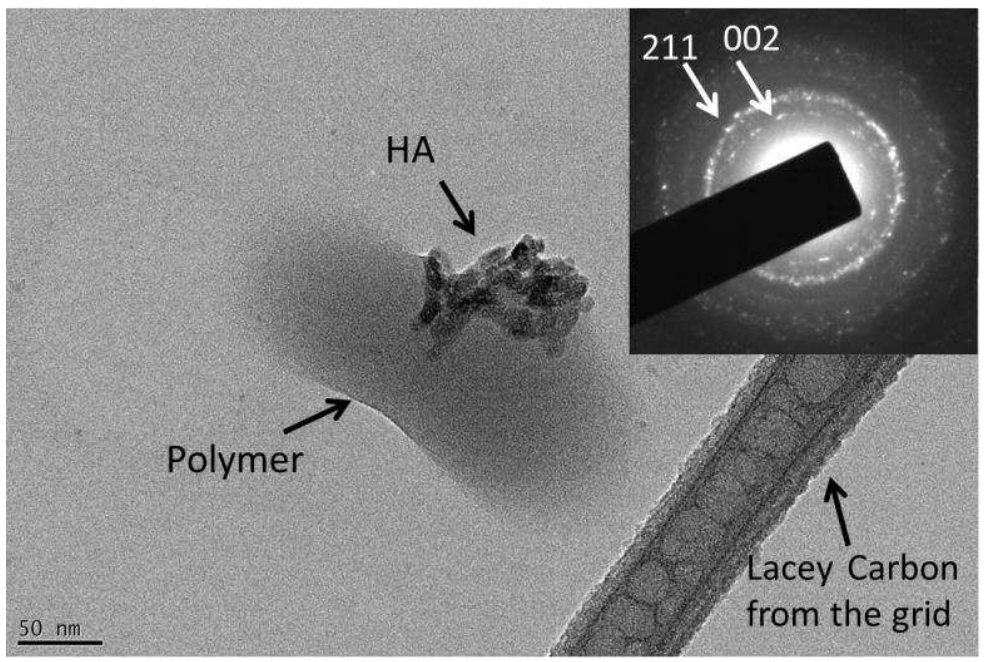

Figure 8. TEM image of a nanoparticle of PLA / PVA / hydroxyapatite. The HA crystal was partially encapsulated by the polymers. The inserted diagram shows electron diffraction from the sample indicating the (002) and (211) planes from hydroxyapatite.

Throughout TEM observations, although the nanoparticles were relatively stable under the electron beam; it is possible that the spherical polymer nanoparticles (as observed by AFM) changed their original morphology immediately after insertion due to the high vacuum environment, of the microscope (around $10^{-7} \mathrm{~Pa}$ ).

\section{Conclusion}

The PLA coated nano-hydroxyapatite is shown to be a great prototype of PLA-nano-hydroxyapatite. Its use for human purposes may be increased by the use of PLA alongside hydroxyapatite. Certainly for human use, there are many possibilities - especially in association with radioisotopes - as demonstrated previously, when the group labelled HA with technetium-99m and doped with holmium166.

\section{Conflict of interest}

The authors have no conflicts of interest.

ISSN: 2057-3782 (Online) 


\section{References}

[1] McMahon RE, Wang L, Skoracki R, Mathur AB. Development of nanomaterials for bone repair and regeneration. J Biomed Mater Res B Appl Biomater. 2013; 101(2): 387-397. [CrossRef] [PubMed Abstract]

[2] Nandi SK, Kundu B, Ghosh SK, De DK, Basu D. Efficacy of nano-hydroxyapatite prepared by an aqueous solution combustion technique in healing bone defects of goat. J Vet Sci. 2008; 9(2): 183-191. [CrossRef] [PubMed Abstract]

[3] Wang Z, Li M, Yu B, Cao L, Yang Q, Su J. Nanocalcium-deficient hydroxyapatite-poly(ecaprolactone)-polyethylene glycol-poly(e-caprolactone) composite scaffolds. Int $J$ Nanomedicine. 2012; 7: 3123-3131. [PubMed Abstract] [PMC Free Article]

Citation: de Souza Albernaz M, Gilberto Weissmuller G, Linhares Rossi A, Malta Rossi A, SantosOliveira R. Polymeric nano-hydroxyapatite coated with polylactic acid (PLA): considering new possibilities for radiopharmacy. Journal of Diagnostic Imaging in Therapy. 2015; 2(1): 9-17.

DOI: http://dx.doi.org/10.17229/jdit.2015-0210-012

Copyright: (C) 2015 Santos-Oliveira R, et al. This is an open-access article distributed under the terms of the Creative Commons Attribution License, which permits unrestricted use, distribution, and reproduction in any medium, provided the original author and source are cited.

Received: 27 January 2015 | Revised: 09 February 2015 | Accepted: 10 February 2015

Published Online 10 February 2015 http://www.openmedscience.com 